\title{
Resolving the Dusty Circumstellar Structure of the Enigmatic Symbiotic Star CH Cygni with the MMT Adaptive Optics System ${ }^{1}$
}

\author{
Beth A. Biller ${ }^{1}$, Laird M. Close ${ }^{1}$, Aigen Li², Massimo Marengo ${ }^{3}$, John H. Bieging ${ }^{1}$, Phil M. \\ Hinz $^{1}$, William F. Hoffmann ${ }^{1}$, Guido Brusa ${ }^{1}$, and Doug Miller ${ }^{1}$ \\ ${ }^{1}$ Steward Observatory, University of Arizona, Tucson, AZ 85721; bbiller@as.arizona.edu \\ ${ }^{2}$ Department of Physics and Astronomy, University of Missouri, Columbia, MO 65211 \\ ${ }^{3}$ Harvard-Smithsonian Center for Astrophysics, 60 Garden Street, Cambridge, MA 02138
}

\begin{abstract}
We imaged the symbiotic star $\mathrm{CH}$ Cyg and two PSF calibration stars using the unique $6.5 \mathrm{~m}$ MMT deformable secondary adaptive optics system. Our highresolution $\left(\mathrm{FWHM}=0.3^{\prime \prime}\right)$, very high Strehl $(98 \pm 2 \%)$ mid-infrared (9.8 and $11.7 \mu \mathrm{m}$ ) images of $\mathrm{CH}$ Cyg allow us to probe finer length scales than ever before for this object. $\mathrm{CH}$ Cyg is significantly extended compared to our unresolved PSF calibration stars ( $\mu$ UMa and $\alpha$ Her) at 9.8 and $11.7 \mu \mathrm{m}$. We estimated the size of the extension by convolving a number of simple Gaussian models with the $\mu$ UMa PSF and determining which model provided the best fit to the data. Adopting the Hipparcos distance for this object of $270 \mathrm{pc}$, we found a nearly Gaussian extension with a FWHM at $9.8 \mu \mathrm{m}$ of $\sim 40.5 \pm 2.7 \mathrm{AU}\left(0.15 \pm 0.01^{\prime \prime}\right)$ and a FWHM at $11.7 \mu \mathrm{m}$ of $45.9 \pm 2.7 \mathrm{AU}\left(0.17 \pm 0.01^{\prime \prime}\right)$. After subtracting out the Gaussian component of the emission (convolved with our PSF), we found a faint $\sim 0.7^{\prime \prime}$ asymmetric extension which peaks in flux $\sim 0.5^{\prime \prime}$ north of the stars. This extension is roughly coincident with the northern knotlike feature seen in HST WFPC2 images obtained in 1999.
\end{abstract}

Subject headings: instrumentation: adaptive optics - binaries: general - stars: evolution — stars: formation — stars: Symbiotic — stars: individual (CH Cygni) - infrared: stars

\footnotetext{
${ }^{0}$ The results presented here made use of the of MMT Observatory, a facility jointly operated by the University of Arizona and the Smithsonian Institution.
} 


\section{Introduction}

Symbiotic stars are a class of interacting binaries consisting of a hot component (usually a white dwarf) which ionizes the stellar wind of a cool component (generally a red giant or Mira, see Kenyon 2000). With typical component separations of $\sim 1-50$ AU, these complex systems are responsible for some of the most dramatic Galactic jet sources (Eyres et al. 2002).

$\mathrm{CH}$ Cyg is one of the most enigmatic symbiotic stars - previous to 1963, CH Cyg showed no variable behavior and was actually used as a M6III spectral type calibrator (see Kenyon 2001, for background details on CH Cyg's properties). Since 1963, CH Cyg has gone through multiple outbursting phases, producing bipolar radio jets in 1984 (Taylor et al. 1986), 1992 (Karovska et al. 1998), and 1998 (Crocker et al. 2001), as well as X-ray jets (Galloway \& Sokoloski 2004), and large ionized outflows $\left(\sim 10^{\prime \prime}\right.$ in size, see Eyres et al. 2002; Corradi et al. 2001). B band flickering studies also point to the existence of an unstable accretion disk around the hot white dwarf component (Sokoloski \& Kenyon 2003a,b).

The CH Cyg system contains at least one red giant/white dwarf pair (Mikolajewski et al. 1987). Currently, there is ongoing debate (Sokoloski \& Kenyon 2003b) as to whether the $\mathrm{CH}$ Cyg system is binary or the only known symbiotic triple star (consisting of either a red giant/white dwarf pair with a G star companion (Hinkle et al. 1993) or a red giant/white dwarf pair with an additional red giant companion (Skopal et al. 1996)). For the purposes of this paper, we adopt the interpretation of the $\mathrm{CH}$ Cyg system as an inner red giant/white dwarf pair with a period of 756 days (Hinkle et al. 1993) and an outer red giant with a period of 14.5 years (Skopal et al. 1996). All three stars are viewed nearly edge on and the outer giant periodically eclipses the inner binary.

As an unresolved triple system, CH Cyg is very difficult to study. In order to completely understand the system it is necessary to disentangle the contribution of each component to the behavior of the system as a whole. Most studies of $\mathrm{CH}$ Cyg focus on the contribution of the hot white dwarf component to the system - jets (Galloway \& Sokoloski 2004), ionized outflows (Eyres et al. 2002; Corradi et al. 2001), and hot component disk flickering (Sokoloski \& Kenyon 2003a,b) primarily trace the properties of the hot component and the matter accreting on to it. In fact, the hot component dominates the total source properties at many wavelengths - to study the cool components, it is important to find a wavelength at which the cool components dominate over the hot component. To this end, we chose to study $\mathrm{CH}$ Cyg at mid-infrared (mid-IR) wavelengths, where the contribution of the hot component is negligible ( $<5 \%$ of the total emission, even during active periods). While near-IR and mid-IR photometry has previously been undertaken for $\mathrm{CH}$ Cyg (Taranova \& Shenavrin 2000, 2004; Bogdanov \& Taranova 2001), CH Cyg has not previously been resolved at these 
wavelengths, since sufficient spatial resolution at mid-IR wavelengths has not been available until recently (Close et al. 2003, Biller et al. 2005). Using the novel technique of adaptive optics at mid-IR wavelengths, we can directly image the emission from the cool component of the system by itself for the first time and study any circumstellar material on finer spatial scales than before.

\section{Observations and Data Reduction}

Data were taken on the night of 2003 May 13 (UT) at the $6.5 \mathrm{~m}$ MMT using the adaptive secondary mirror AO system (Wildi et al. 2003) with the BLINC-MIRAC3 camera (Hoffman et al. 1998; Hinz et al. 2000). Currently all other existing AO systems use a warm reimaged adaptive mirror and require several additional warm optical surfaces between the secondary and the detector. With the unique MMT adaptive secondary, we remove many of these warm optical surfaces from the optical path compared to a standard AO system. This results in a considerably reduced thermal background at the mid-IR, thus making adaptive optics possible for mid-IR observations. The adaptive secondary corrected the first 52 system modes and achieved Strehl ratios as high as $0.97 \pm 0.03$ from 8.8 to $18 \mu \mathrm{m}$. These Strehl ratios are the highest ever presented in the literature for a large ground based telescope (Close et al. 2003, Biller et al. 2005). For details on the MMT Adaptive Secondary AO system, see Wildi et al. (2003). We utilized the $128 \times 128$ SiAs BIB 2-20 $\mu$ m MIRAC3 camera (Hoffmann et al. 1998). The $0.088^{\prime \prime} /$ pixel scale was used with the 9.8 and $11.7 \mu \mathrm{m} 10 \%$ bandwidth filters. To remove thermal and detector instabilities we chopped at $1 \mathrm{~Hz}$ with an internal pupil-plane cold chopper in the interface dewar BLINC (Hinz et al. 2000) between the AO system and MIRAC3.

We observed with the AO system locked continuously on $\mathrm{CH}$ Cyg. The $15^{\circ}$ tilted BLINC dewar window is a high quality dichroic which reflected the visible light $(\lambda<1 \mu \mathrm{m})$ to the AO wavefront sensor and transmitted the IR through BLINC to MIRAC3. Since the internal chopper in BLINC was past the dichroic, continuous $1 \mathrm{~Hz}$ chopping did not affect the visible light beam and hence the AO lock was unaffected. To further calibrate the background (in addition to chopping) we nodded $\sim 6-8^{\prime \prime}$ in the telescope's azimuth direction (the horizontal direction in Figure 1) every minute. The internal chopper was set to run in the altitude direction (the vertical direction) with a chop throw of $\sim 20^{\prime \prime}$. The derotator was disabled during these observations to help minimize the residual background structure.

The $0.505^{\prime \prime} \mathrm{PA}=269^{\circ}$ Washington Double Star catalog astrometric binary WDS 02589+2137 BU was observed during a later run (2003, November 25 UT) and used to calibrate the camera's orientation and its $0.088^{\prime \prime} /$ pixel platescale. 
For the 9.8 and $11.7 \mu \mathrm{m}$ filters we obtained $2 \times 1$ minute coadded chop differenced images (one image from each nod) for $\mathrm{CH}$ Cyg. We utilized a custom IRAF script to reduce this mid-IR data (Biller et al. 2005). The script produced two background subtracted images by subtracting the image taken at the first nod position with that taken at the second, and vice versa. Images were then bad pixel corrected, flat-fielded, cross-correlated, and aligned to a reference nod image (to an accuracy of $\sim 0.02$ pixels). Mid-IR images of the PSF calibration stars ( $\mu$ UMa and $\alpha$ Her, both of these stars are binary but appear unresolved to MIRAC, see Close et al. [2003] for a discussion of PSF suitability) were obtained and reduced in an identical manner to CH Cyg. Reduced images of CH Cyg and the two PSF stars are presented in Figure 1. In order to directly compare our science images with the PSF images, we have not rotated the images to place north up - azimuth is vertical and altitude is horizontal. At the time of the observations, the Parallactic Angle (PA) was roughly $160^{\circ}$.

The CH Cyg images appear slightly yet significantly extended compared to images of the two PSF stars. To highlight this extension, we present one-dimensional normalized cut plots in both altitude and azimuth directions across the peak for the 9.8 and $11.7 \mu \mathrm{m}$ images of CH Cyg and PSF star $\mu$ UMa in Figures 2 and 3. Three rows/columns were averaged together for each cut plot to increase signal to noise. Since $\mathrm{CH}$ Cyg is a very bright object (565 Jy at $12 \mu \mathrm{m}$ ) and errors are dominated by photon noise, error bars for these plots are considerably smaller than the size of the plot symbols and have not been plotted. In all the altitude and azimuth cutplots at 9.8 and $11.7 \mu \mathrm{m}$, the $\mathrm{CH}$ Cyg profiles are noticeably extended compared to those for the $\mu$ UMa PSF. We measured the FWHM, ellipticity, and positional angle of any such ellipticity for all the images in Figure 1, as well as for images of RV Boo, an AGB star with a small extended dust disk observed the same night (Biller et al. 2005). In Figure 4 we plot the ellipticity and FWHM for our datasets. CH Cyg has a similar eccentricity but a significantly higher FWHM than the PSF stars. Thus, CH Cyg looks clearly extended compared to the PSF stars.

In order to detect the gas counterpart to the mid-IR emitting dust, CH Cyg was observed in the $\mathrm{CO}(1-0)$ transition at the Arizona Radio Observatory $12 \mathrm{~m}$ telescope on Kitt Peak on the night of 29 June 2005. No CO (1-0) was detected in 4 hours of integration time.

\section{Analysis}

In order to study small-scale circumstellar structure around $\mathrm{CH} \mathrm{Cyg}$, we deconvolved our high $\mathrm{S} / \mathrm{N}(\mathrm{S} / \mathrm{N}>1000)$ images of $\mathrm{CH}$ Cyg using both the PSF stars $\mu$ UMa and $\alpha$ Her (see Biller et al. 2005, for details on PSF star selection) with the IRAF Lucy deconvolution task (Lucy 1974). So as not to introduce noise amplification artifacts due to deconvolution, 
we deconvolved images for only 10 iterations. To test whether artifacts were being introduced by the Lucy algorithm, we also deconvolved images of the $\mu$ UMa PSF with those of the $\alpha$ Her PSF in order to establish the expected result of deconvolving one point source by another point source. Deconvolved images of CH Cyg and the PSF stars appear in Figure 5. Due to the very high Strehl $(\sim 0.98)$ and high signal-to-noise ratio in our PSF images we could detect low contrast structure on "super-resolution" scales of $\sim 0.2$ " with the IRAF Lucy deconvolution task (Biller et al. 2005). After deconvolution, CH Cyg still appears significantly extended compared to the deconvolved PSF images.

To estimate the size of the extension around $\mathrm{CH}$ Cyg, we convolved a range of simple Gaussian dust envelope models (with FWHMs of 0.1, 0.14, 0.15, 0.16, 0.18, and 0.2" before convolution) with the $\mu$ UMa PSF. We determined the best fit model by comparing model cut plots with data cut plots; the best fit model minimizes the standard deviation of [cutplot(data) - cutplot(model)]. At $9.8 \mu \mathrm{m}$, the $0.15^{\prime \prime}$ model provided the best fit to the altitude cut plot, while the $0.14^{\prime \prime}$ model provided the best fit to the azimuth cut plot. At $11.7 \mu \mathrm{m}$, the $0.17^{\prime \prime}$ model provided the best fit to the altitude cut plot, while the $0.16^{\prime \prime}$ model provided the best fit to the azimuth cut plot. Best fit model cut plots are presented in Figures 2,3. The slightly broader cut plot profile along the altitude direction in both these cases is probably due to instrumental effects and is not physical. Utilizing the Hipparcos distance of $\sim 270 \mathrm{pc}$, we adopt a FWHM at $9.8 \mu \mathrm{m}$ of $\sim 40.5 \pm 2.7 \mathrm{AU}\left(0.15 \pm 0.01^{\prime \prime}\right)$, and a FWHM at $11.7 \mu \mathrm{m}$ of $45.9 \pm 2.7 \mathrm{AU}\left(0.17 \pm 0.01^{\prime \prime}\right)$.

We scaled and subtracted the best fit Gaussian model (convolved with the $\mu$ UMa PSF) from the data in order to reveal any asymmetric structure. Images of residual asymmetric structure are presented in Figure 6. The residual structure peaks in intensity $\sim 0.5^{\prime \prime}$ north of the star (marked by a diamond) and is roughly coincident with the extension seen by Crocker et al. (2001) at $\sim 0.7^{\prime \prime}$ north of the star in HST WFPC2 images (marked by the letter A in Fig. 6). The structure also appears to be extended along a similar axis to the radio/optical jets.

\section{Modeling}

Since the residual asymmetric structure we observe around $\mathrm{CH}$ Cyg is faint compared to the symmetric Gaussian structure (peak fluxes only 2\% that of the symmetric component) we have concentrated our modeling efforts on the symmetric component only. We model the IR emission of $\mathrm{CH}$ Cyg as an optically thin shell passively heated by the star.

We approximate the stellar photosphere by the Kurucz (1979) model spectrum for 
M6 IIIe stars with an effective temperature of $T_{\text {eff }}=3000 \mathrm{~K}$. The dust is taken to be amorphous silicate since $\mathrm{CH}$ Cyg is a $\mathrm{M}$ star. Taking all grains to be spherical with $a$ being their spherical radius, we assume a power-law dust size distribution $d n(a) / d a \propto a^{-\alpha}$ which is characterized by a lower-cutoff $a_{\text {min }}$, upper-cutoff $a_{\max }$ and power-law index $\alpha$. For simplicity, we take $a_{\min }=0.01 \mu \mathrm{m}$ since smaller grains will undergo single-photon heating, and $a_{\max }=1000 \mu \mathrm{m}$ since larger grains are not well constrained by the currently available IR photometry.

We approximate the dust spatial distribution with a modified power-law $d n / d r \propto$ $\left(1-r_{\min } / r\right)^{\beta}\left(r_{\min } / r\right)^{\gamma}$ where $r_{\min }$ is the inner boundary of the envelope which we take to be the location where silicate dust sublimates. For CH Cyg with a luminosity $L_{\star} \approx 6900 L_{\odot}$, submicron-sized silicate dust achieves an equilibrium temperature of $T \approx 1500 \mathrm{~K}$ and starts to sublimate at $r \approx 2 \mathrm{AU}$. Therefore we take $r_{\min }=2 \mathrm{AU}$. This functional form has the advantage that on one hand, it behaves like a power-law $d n / d r \propto r^{-\gamma}$ at larger distances $\left(r \gg r_{\min }\right)$, and on the other hand it peaks at $r_{\mathrm{p}}=r_{\min }(\beta+\gamma) / \gamma$, unlike the simple powerlaw which peaks at $r_{\text {min }}$. The latter is unphysical since one should not expect dust to pile up at $r_{\text {min }}$ where dust sublimates! We take $\gamma=2$ as expected from a stationary outflow with a constant mass loss rate (e.g. see Habing, Tignon, \& Tielens 1994). But other $\gamma$ values have also been considered (see below), in view of the time-variable nature of this object. We take $r_{\max }=200 \mathrm{AU}$ which is large enough for our IR emission modeling purpose since there is very little dust beyond this outer boundary which emit at the wavelengths we are interested $(\lambda \lesssim 200 \mu \mathrm{m})$. Therefore, we have only two free parameters: $\alpha$ - the power-law exponent for the dust size distribution and $\beta$ - the dust spatial distribution parameter which determines where the dust peaks.

Using the dielectric functions of "astronomical silicates" (Draine \& Lee 1984) and Mie theory (Bohren \& Huffman 1983), we calculate the absorption cross sections of amorphous silicate grains as a function of size, as well as their equilibrium temperatures as a function of radial distance from the central star (in thermal equilibrium with the illuminating starlight). We then obtain the dust model IR emission spectrum by integrating the dust emission over the entire size range and the entire envelope.

The model spectrum is then compared with (1) the $9.8 \mu \mathrm{m}$ and $11.7 \mu \mathrm{m}$ photometry from our MMT AO images, (2) the 12, 25, 60, and $100 \mu \mathrm{m}$ IRAS (Infrared Astronomical Satellite) broadband photometry, (3) the $7.7-22.7 \mu \mathrm{m}$ mid-IR low-resolution spectroscopy obtained with the IRAS Low Resolution Spectrometer (LRS; with a resolution of $\lambda / \Delta \lambda \approx 20$ ), (4) the 2.5-45 $\mu \mathrm{m}$ near- to mid-IR high-resolution spectroscopy obtained with the Short Wavelength Spectrometer (SWS; $\lambda / \Delta \lambda \approx 2000$ ) on board the Infrared Space Observatory (ISO), and (5) the $43-197 \mu \mathrm{m}$ mid- to far-IR medium-resolution spectroscopy obtained with the ISO Long 
Wavelength Spectrometer (LWS; $\lambda / \Delta \lambda \approx 150-200$ ). Given that CH Cyg is a variable star, it is to our great surprise that these observational data taken at different time epochs are in reasonably good agreement with each other: the MMT-AO and IRAS fluxes are only $\sim 12 \%$ and $\sim 25 \%$, respectively, smaller than the ISO SWS flux (see Fig. 7). The SWS spectrum is also in close agreement with the $\mathrm{K}, \mathrm{L}$, and M photometry. But the IRAS LRS flux is lower than the SWS spectrum by a factor of $\sim 16$. We therefore take the ISO SWS and LWS spectra as the comparison basis and increase the MMT-AO fluxes, the IRAS broadband fluxes, and the IRAS LRS spectrum by a factor of 1.12, 1.25 and 16, respectively. This brings the MMT-AO and IRAS data into agreement with the ISO data.

The model which best fits the observed IR emission spectrum and photometric points has $\alpha \approx 4.1$ and $\beta \approx 10$ (see Fig. 7 ), with the dust distribution peaking at $r_{\mathrm{p}} \approx 12 \mathrm{AU}$, a total visual optical depth $\tau_{V} \approx 0.16$, and a total dust mass $m_{\text {dust }} \approx 1.04 \times 10^{27} \mathrm{~g} \approx 5.2 \times 10^{-7} M_{\odot}$. It is not easy to translate the total dust mass into the mass loss rate which is actually considered as one of the most important tasks in the study of stellar evolution (e.g. see Willson 2000). To estimate the mass loss rate of $\mathrm{CH}$ Cyg, we need to assume a gas-to-dust mass ratio $m_{\text {gas }} / m_{\text {dust }}$ and a gas outflow velocity $v$. With $m_{\text {gas }} / m_{\text {dust }}=100$ and $v=50 \mathrm{~km} \mathrm{~s}^{-1}$ (based on the velocity of the Ca-II absorption component which, according to Skopal et al. 1996, is likely associated to the expanding circumstellar wind), we estimate the mass loss rate of $\mathrm{CH}$ Cyg to be $\approx 2.7 \times 10^{-6} M_{\odot} \mathrm{yr}^{-1}$.

In order to compare the best fit model directly to our MMT AO images, we convolved the model radial profile with our AO PSF image of $\mu$ UMa. After convolution, the best fit model to the observed IR emission spectrum and photometric points produces an image appreciably larger than the MMT AO image. We have considered models with a grid of parameters $\left(\beta=2-20, \gamma=1-4, r_{\max }=25-250 \mathrm{AU}\right)$. While multiple models fit the IR emission spectrum reasonably well, none of the models is able to simultaneously replicate the AO image and the observed IR emission. For instance, the best fit model to the IR emission spectrum $(\alpha \approx 4.1$ and $\beta \approx 10$ ) has a FWHM $\sim 0.4$ " at $9.8 \mu \mathrm{m}$ (after convolution with the AO PSF), while the AO image of CH Cyg has a FWHM $<0.37$ ". Given the extremely high Strehl ratios during these observations $(\sim 100 \%)$ and the resultant high image fidelity, this is a significant divergence! These models are likely too simple to fully describe this complex system. The models assume only one star, with a steady state outflow. However, the $\mathrm{CH}$ Cyg system contains 3 stars - we have not taken into account the disruptive effects upon the dust of the other two stellar components of this system. The assumed functional form for the dust spatial distribution may be too simplified. We should also note that, as a variable star, the spatial and size distributions of the dust (which determine the surface brightness distribution of the dust shell around $\mathrm{CH} \mathrm{Cyg}$ ) at the epoch when the AO images were taken can be quite different from those when the IRAS photometry and the ISO spectroscopy were 
obtained. As a result, it is very unlikely that a dust model will fit both the observed IR emission spectrum and the AO images. Thus this is not a test of the validity of the model.

\section{Discussion}

Circumstellar dust in the CH Cyg system has previously been studied by Taranova and collaborators. With near- and mid-IR photometric observations, Taranova \& Shenavrin (2004) follow the properties of a circumstellar dust envelope around CH Cyg. According to Taranova \& Shenavrin (2004)'s model, mass was injected into the cool component's circumstellar dust envelope around 1983 by an as yet unknown mechanism, coincident with the bipolar radio outflow observed by Taylor et al. (1986). The optical depth of the dust envelope increased through 1992. By 1996, the dust envelope began to dissipate and had decreased to pre-mass injection optical depths by 2001.

Taranova \& Shenavrin $(2000,2004)$ model the circumstellar dust envelope of CH Cyg as a single temperature shell around the cool component which varies in size and total dust mass during mass injection episodes. From 1983 to 2003, they claimed that the dust shell radius varied between $\sim 10^{14}-10^{15} \mathrm{~cm}$, or from about $7-70 \mathrm{AU}$ (but Bogdanov \& Taranova [2001] argue for a much large extension: 15-15000 AU). We observe CH Cyg to be similarly extended, with a FWHM of $\sim 40-46$ AU.

The symmetric extended dust structure around $\mathrm{CH}$ Cyg is considerably larger than the orbits of the stellar components. In order to estimate the semimajor axis of the inner 756 day period binary, we adopt a white dwarf/hot component mass of $\sim 0.2 M_{\odot}$ and a red giant/cool component mass of $\sim 2 M_{\odot}$ (Hinkle et al. 1993). We calculate a semimajor axis estimate of $\sim 2$ AU. To estimate the semimajor axis of the outer 14.5 year period binary, we adopt an outer red giant mass of $\sim 2 M_{\odot}$ and find a semimajor axis estimate of $\sim 10$ AU. The observed dust envelope extension of $\sim 40-46 \mathrm{AU}$ is larger than both the inner and outer binary orbits. Thus, the dust envelope contains all three stellar components of this system. The fact that all three stellar components of this system lie within the dust envelope may be significant in explaining why our AO dust shell images diverge from our spherically symmetric dust envelope models - possibly, the stellar components of this system have a hand in shaping the dust envelope into something other than a classic, red giant wind spherical shell. However, whatever specific contribution the stellar components have had on the shape of the dust envelope remains unresolved and unquantified.

After subtracting out the symmetric component of the emission, the residual emission appears extended along a similar north-south axis to those of the radio jets reported by 
Crocker et al. (2001). The residual structure peaks in flux $\sim 0.5^{\prime \prime}$ north of the star and is roughly coincident to the knotlike structure observed by Crocker et al. (2001) at 0.7" north of the star in HST WFPC2 images (marked by the letter A in Fig. 6). The structure also appears to be extended along a similar axis to the radio/optical jets, and is similar to the extent of the radio jets and ionized optical structure. However, while bipolar structure is observed in both the radio and optical, the mid-IR structure is unipolar. One possible explanation is that mid-IR emitting dust is denser towards the northern lobe of the bipolar outflow. The outflow shocks and heats the dust towards the north, hence a bright mid-IR dust structure is observed. Towards the south, the outflow is unimpeded by dust and, thus, has a larger optical and radio extent, but no mid-IR counterpart.

\section{Conclusions}

The combination of adaptive optics with a deformable secondary produces very high Strehl images in the mid-IR and high PSF stability regardless of the seeing, airmass, or target brightness. Such PSF stability allows us to determine object properties with very high fidelity. Adopting the Hipparcos distance to $\mathrm{CH}$ Cyg of $\sim 270 \mathrm{pc}$, we found a nearly spherically symmetric extension with a FWHM at $9.8 \mu \mathrm{m}$ of $\sim 40.5 \pm 2.7 \mathrm{AU}\left(0.15 \pm 0.01^{\prime \prime}\right)$ and a FWHM at $11.7 \mu \mathrm{m}$ of $\sim 45.9 \pm 2.7 \mathrm{AU}\left(0.17 \pm 0.01^{\prime \prime}\right)$. After subtracting out a model of the spherically symmetric emission, we found residual extension $\sim 0.5^{\prime \prime}$ north of the star roughly coincident with the optical knotlike feature observed by Crocker et al. (2001).

Adaptive optics at mid-IR wavelengths is a very promising new technique that allows for uniquely stable PSFs and high Strehls. A high degree of PSF stability will eliminate morphological ambiguities due to poor (seeing-limited) PSF calibrations. Mid-IR AO should have a significant impact on any field where mid-IR imaging is possible.

These MMT observations were made possible with the hard work of the entire Center for Astronomical Adaptive Optics (CAAO) staff at the University of Arizona. Graduate student Wilson Liu helped run the MIRAC3 camera during the run. The adaptive secondary mirror is a joint project of University of Arizona and the Italian National Institute of Astrophysics - Arcetri Observatory. We would also like thank the whole MMT staff for their excellent support and flexibility during our commissioning run at the telescope.

The secondary mirror development could not have been possible without the support of the Air Force Office of Scientific Research under grant AFOSR F49620-00-1-0294. BAB is supported by the NASA GSRP grant NNG04GN95H and NASA Origins grant NNG056L71G. LMC acknowledges support from NASA Origins grant NNG056L71G and 
NSF CAREER award. AL acknowledges support from the University of Missouri Summer Research Fellowship, the University of Missouri Research Board, and the NASA award P20436. JHB acknowledges support from NSF grants AST-9987408 and AST-0307687.

\section{REFERENCES}

Biller, B. A., et al. 2005, ApJ, 620, 450

Bogdanov, M.B., \& Taranova, O.G. 2001, Astronomy Reports, 45, 797

Bohren, C. F., \& Huffman, D. R. 1983, Absorption and Scattering of Light by Small Particles, New York: Wiley

Close, L.M., et al. 2003, ApJ, 598, L35

Corradi, R.L.M., Munari, U., Livio, M., Mampaso, A., Goncalves, D., \& Schwarz, H.E. 2001, ApJ, 560, 912

Crocker, M.M., et al. 2001, MNRAS, 326, 781

Draine, B.T., \& Lee, H.M. 1984, ApJ, 285, 89

Eyres, S.P.S., et al. 2002, MNRAS, 335, 526

Galloway, D.K., \& Sokoloski, J.L. 2004, ApJ, 613, L61

Habing, H.J., Tignon, J., \& Tielens, A.G.G.M. 1994, A\&A, 286, 523

Hinkle, K.H., Fekel, F.C., Johnson, D.S., \& Scharlach, W.W.G. 1993, AJ, 105, 1074

Hinz P.M., et al. 2000, SPIE, 4006, 349

Hoffmann, W. et al. 1998, SPIE, 3354, 647

Karovska, M., Carilli, C.L., \& Mattei, J.A. 1998, Journal of the American Association of Variable Star Observers, 26, 97

Kenyon, S.J. 2000, in The Encyclopedia of Astronomy and Astrophysics, ed. P. Murdin (Bristol: Institute of Physics), 1630

Kenyon, S.J. 2001, in The Encyclopedia of Astronomy and Astrophysics, ed. P. Murdin (Bristol: Institute of Physics), 288

Kurucz, R.L. 1979, ApJS, 40, 1 
Lucy, L.B. 1974, AJ, 79, 745

Mikolajewski, M., Mikolajewska, J., \& Tomov, T. 1987, AP\&SS, 131, 733

Skopal, A., et al. 1996, MNRAS, 327, 346

Sokoloski, J.L., \& Kenyon, S.J. 2003a, ApJ, 584, 1021

Sokoloski, J.L., \& Kenyon, S.J. 2003b, ApJ, 584, 1027

Taranova, O.G. \& Shenavrin, V.I. 2000, Astronomy Reports, 44, 460

Taranova, O.G. \& Shenavrin, V.I. 2004, Astronomy Reports, 48, 813

Taylor, A.R., Seaquist, E.R., \& Mattei, J.A. 1986, Nature 319, 38

Wildi, F.P., Brusa, G., Lloyd-Hart, M., Close, L.M., \& Riccardi, A. 2003, SPIE, 5169, 17

Willson, L. A. 2000, ARA\&A, 38, 573 


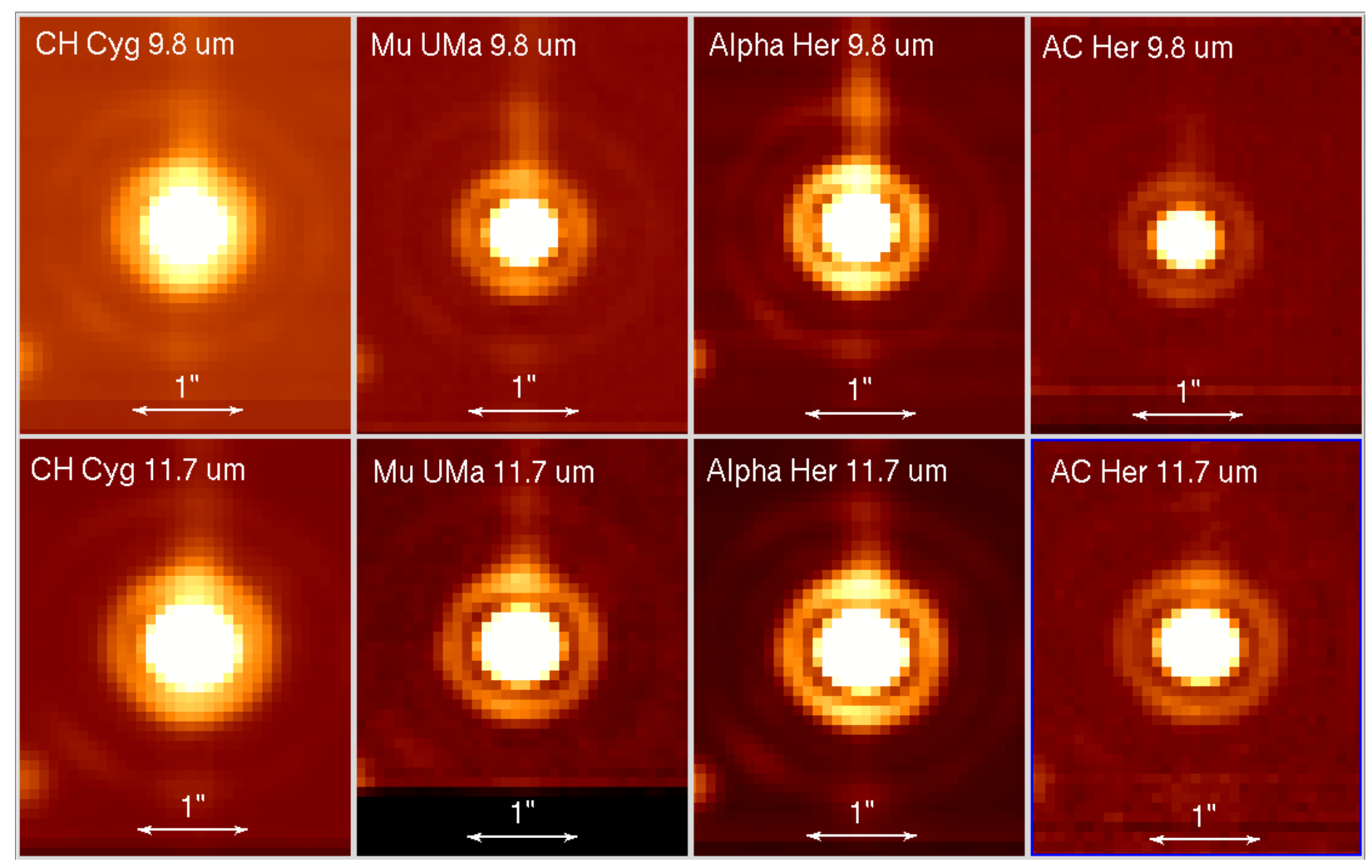

Fig. 1. - The 9.8 and $11.7 \mu \mathrm{m}$ images of CH Cyg and PSF stars $\mu$ UMa, $\alpha$ Her, and AC Her as observed at the MMT. The first, second, and third Airy rings are visible in these images. CH Cyg appears slightly extended compared to the PSF stars - while the PSF stars show a peak distinct from the first Airy ring, the slightly extended emission from $\mathrm{CH}$ Cyg blurs into the first Airy ring. The faint point source in the far lower left of each MMT image is a MIRAC3 ghost. 

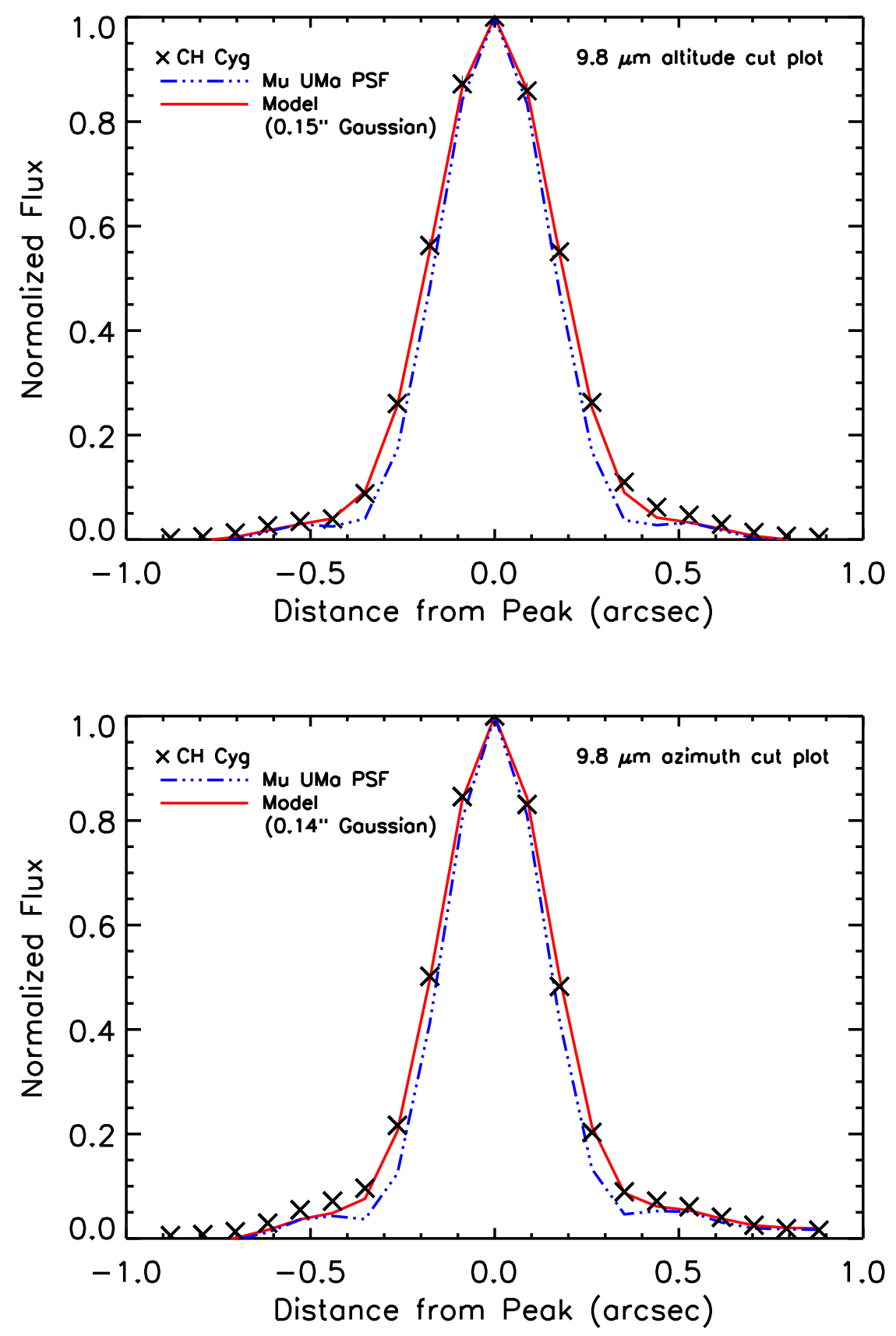

Fig. 2.- One dimensional cut plots along the altitude (top) and azimuth (bottom) directions for the $9.8 \mu \mathrm{m}$ images of $\mathrm{CH}$ Cyg and the PSF star $\mu$ UMa. The CH Cyg data is plotted as crosses, the $\mu$ UMa PSF is plotted as a dotted blue line, and the best fit Gaussian model convolved with the $\mu$ UMa PSF is plotted as a solid red line. Error bars are small compared to the size of the plot symbols. CH Cyg appears slightly but notably extended relative to the $\mu$ UMa PSF. 

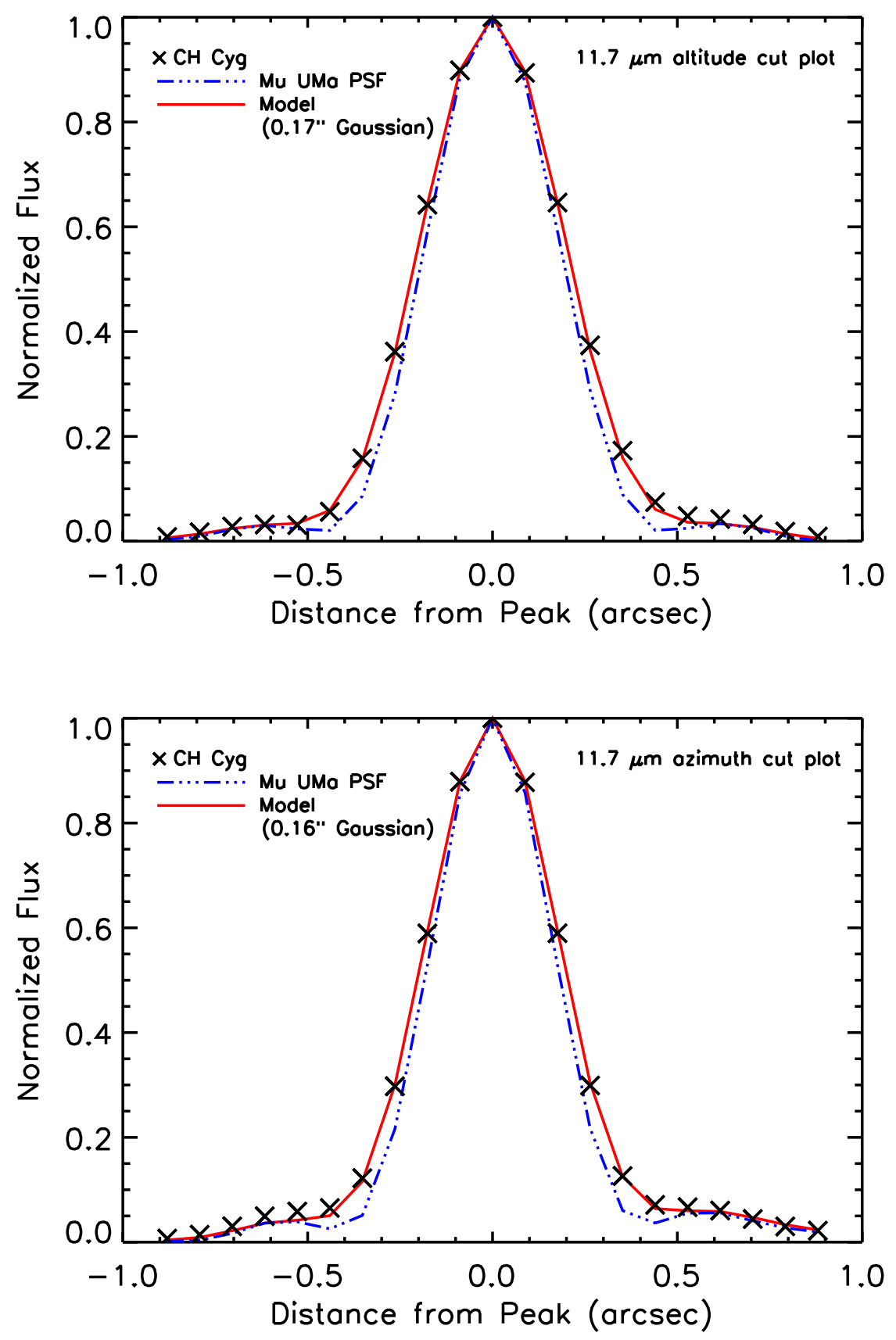

Fig. 3.- One dimensional cut plots along the altitude (top) and azimuth (bottom) directions for the $11.7 \mu \mathrm{m}$ images of $\mathrm{CH}$ Cyg and PSF star $\mu \mathrm{UMa}$. The CH Cyg data is plotted as crosses, the $\mu$ UMa PSF is plotted as a dotted blue line, and the best fit Gaussian model convolved with the $\mu$ UMa PSF is plotted as a solid red line. Error bars are small compared to the size of the plot symbols. CH Cyg appears slightly but notably extended relative to the $\mu$ UMa PSF. 

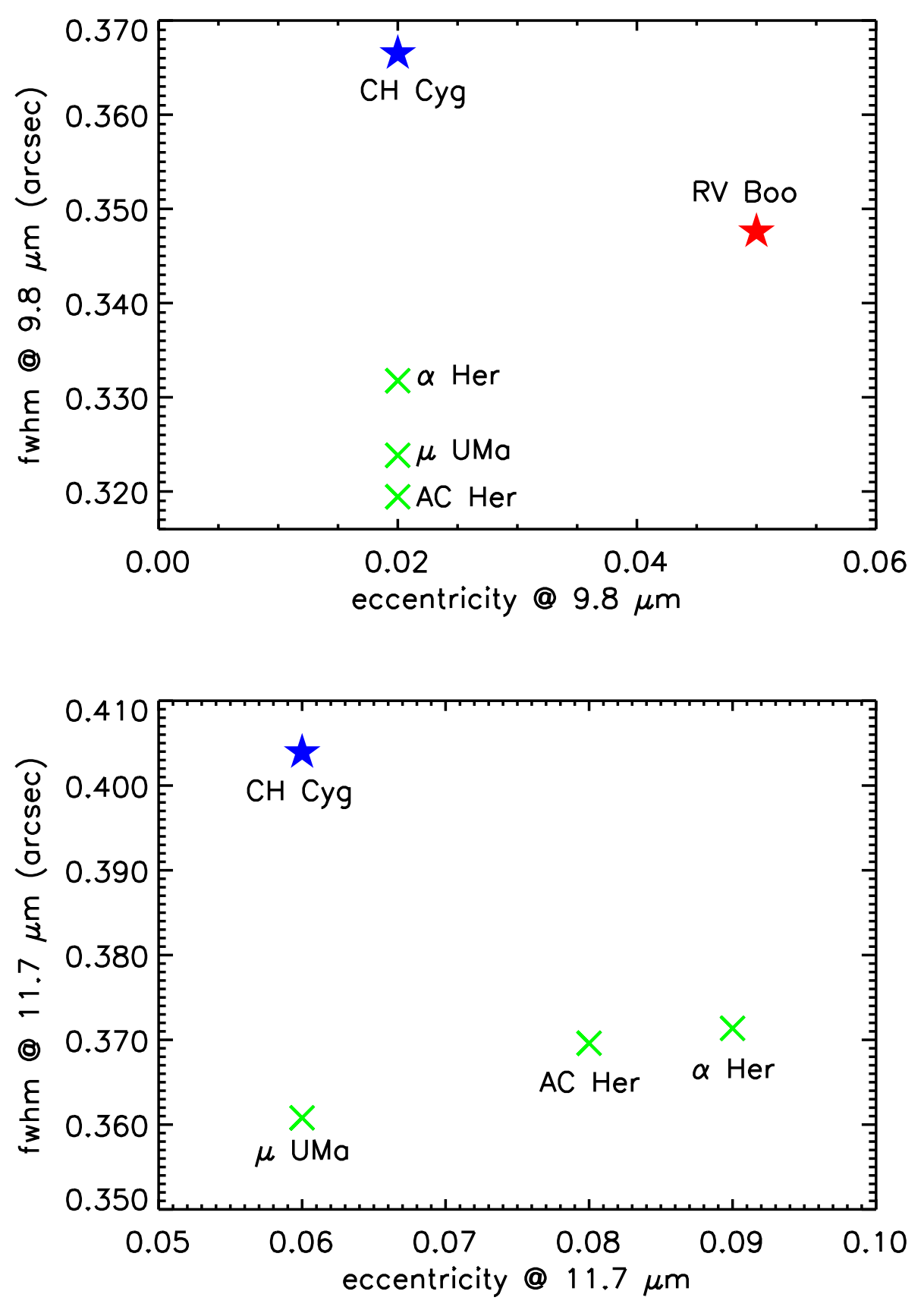

Fig. 4.- The $9.8 \mu \mathrm{m}$ (top) and $11.7 \mu \mathrm{m}$ (bottom) FWHM and ellipticity of CH Cyg and the PSF stars $\mu$ UMa and $\alpha$ Her. CH Cyg appears slightly extended in FWHM compared to the PSF stars. 


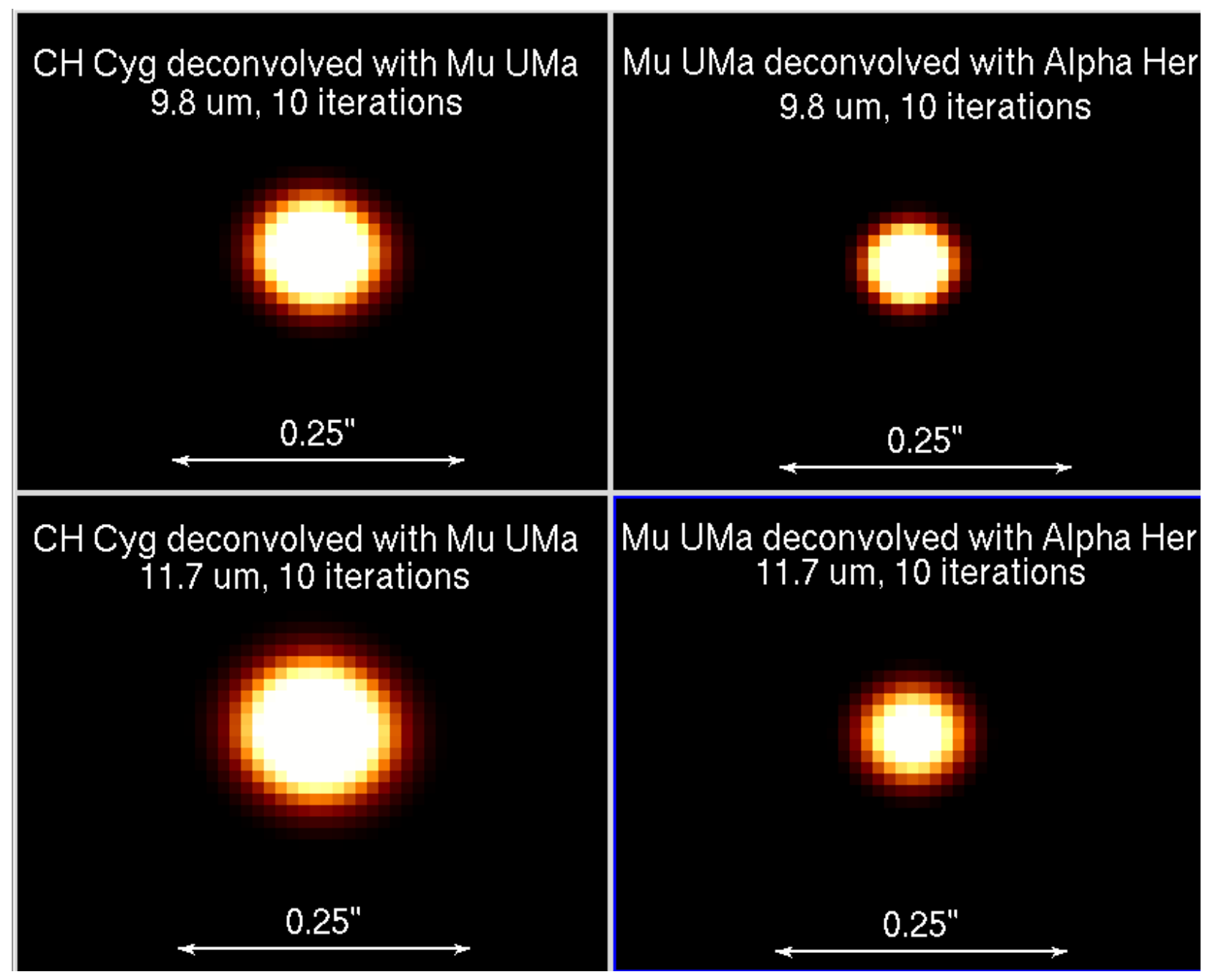

Fig. 5.- Deconvolved images of $\mathrm{CH}$ Cyg. The plate scale (after magnification) is 29.3 mas pixel $^{-1}$. Deconvolved images of the $\mu$ UMa PSF (deconvolved using $\alpha$ Her as a $\mathrm{PSF}$ ) is shown on the right for comparison. CH Cyg appears slightly extended after deconvolution compared to the PSF stars. 

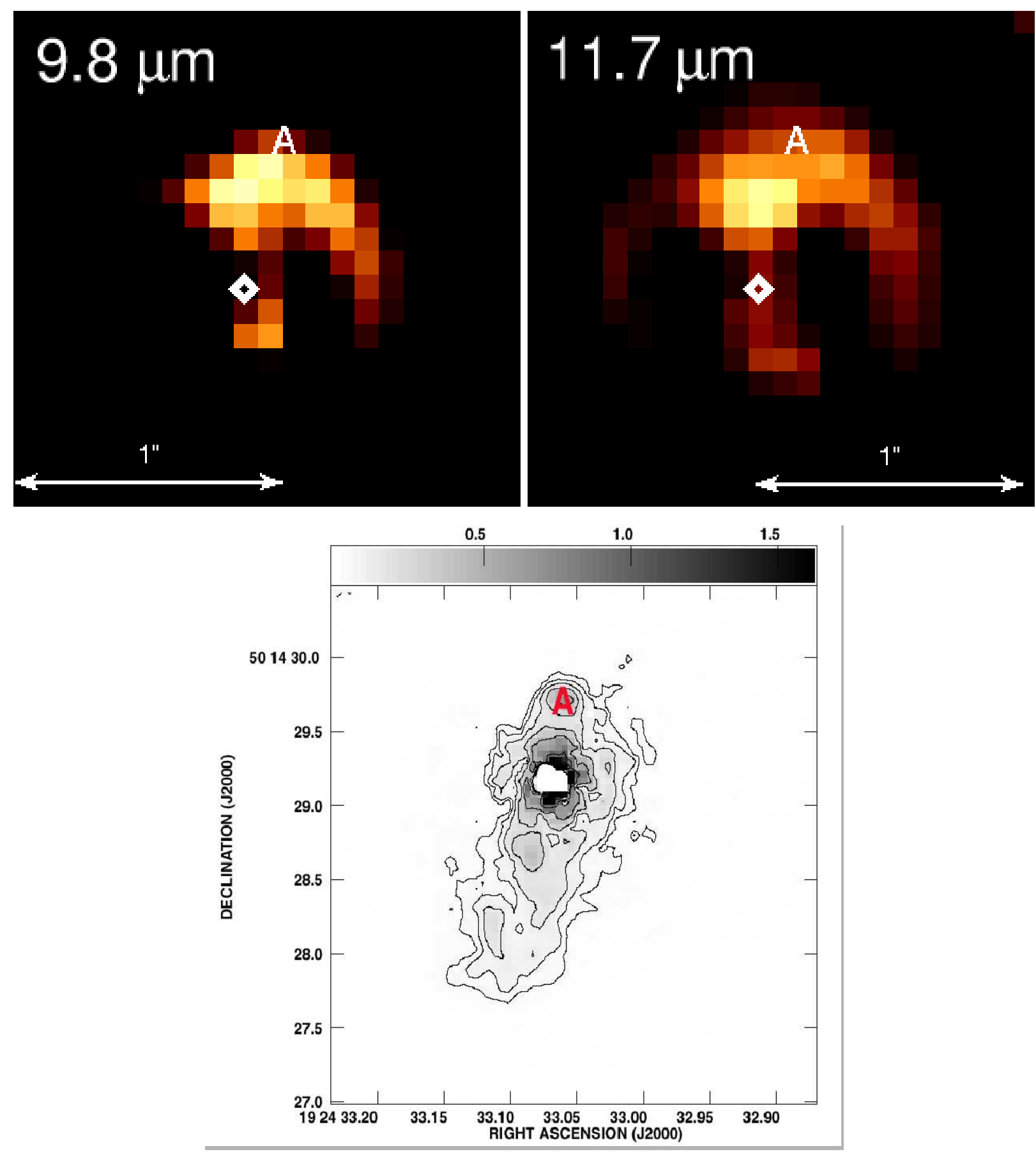

Fig. 6. - Top: Faint residual structure ( $\leq 2 \%$ of the peak) left over after scaling and subtracting the best-fit Gaussian model (convolved with the $\mu$ UMa PSF) from the data. North is up and east is left in these images. The diamond point represents the location of the stellar components of the $\mathrm{CH}$ Cyg system in these images. Bottom: An HST WFPC2 image of CH Cyg from August 1999 (Crocker et al. 2001) is shown for comparison. The residual extension in the mid-IR is roughly coincident with the northern knotlike/bipolar feature observed by Crocker et al. (2001) in the optical and the radio. 


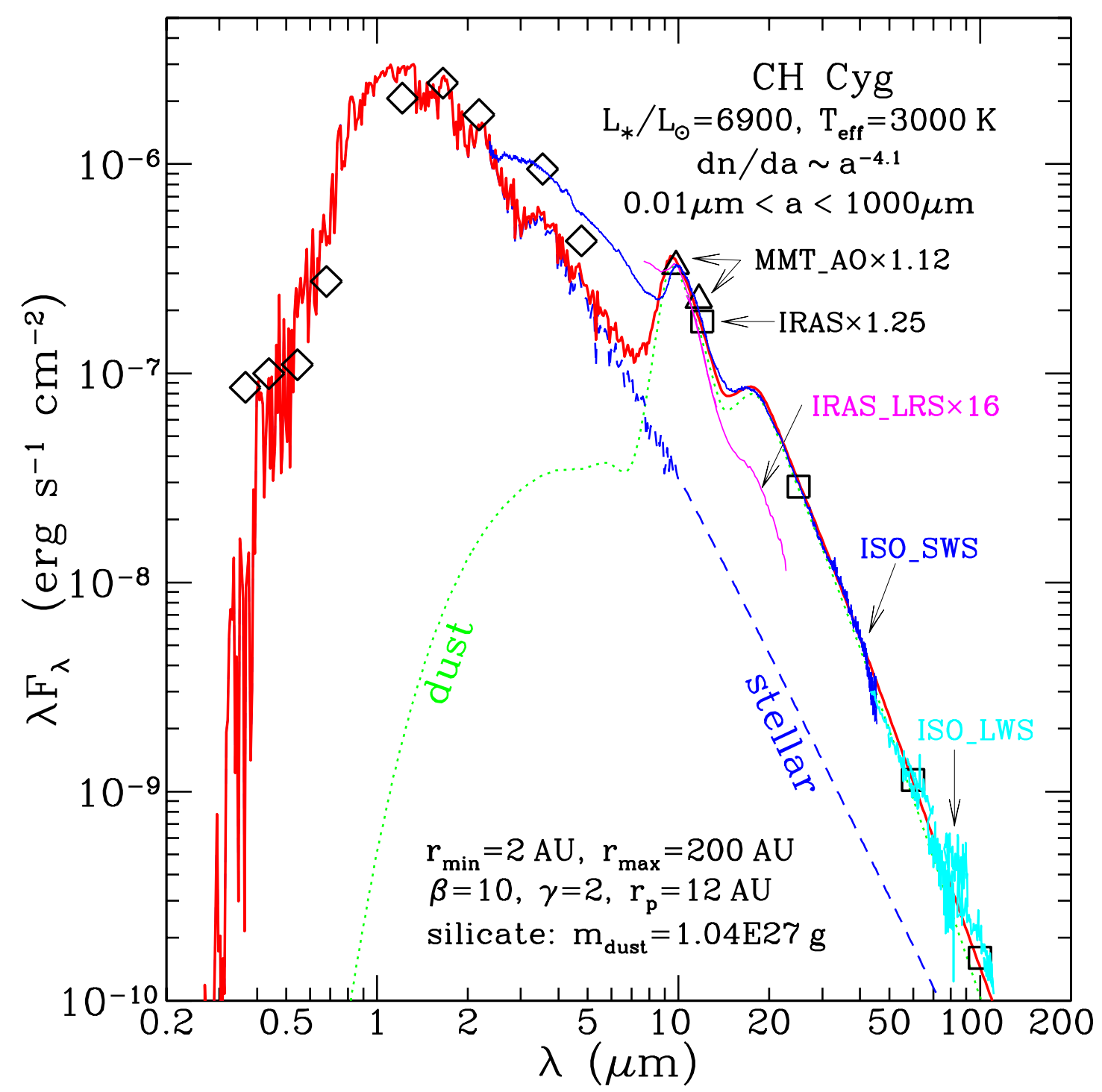

Fig. 7.- IR emission and model fit to the $\mathrm{CH}$ Cyg dust shell. Open black diamonds - U, $\mathrm{B}, \mathrm{V}, \mathrm{R}, \mathrm{J}, \mathrm{H}, \mathrm{K}, \mathrm{L}$, and M photometry from literature; open black triangles - MMT-AO photometry at $9.8 \mu \mathrm{m}$ and $11.7 \mu \mathrm{m}$ (increased by a factor of 1.12); open black squares IRAS photometry at 12, 25, 60 and $100 \mu \mathrm{m}$ (increased by a factor of 1.25). Blue dashed line - stellar photospheric spectrum; solid blue line - ISO SWS spectrum; solid cyan line - ISO LWS spectrum; solid magenta line - IRAS LRS spectrum (increased by a factor of 16). The dust model is shown as a green dotted line, while the solid red line plots the sum of the dust and the star. 